This is the accepted manuscript made available via CHORUS. The article has been published as:

\title{
Origin of Perpendicular Magnetic Anisotropy and Large Orbital Moment in Fe Atoms on MgO
}

S. Baumann, F. Donati, S. Stepanow, S. Rusponi, W. Paul, S. Gangopadhyay, I. G. Rau, G. E. Pacchioni, L. Gragnaniello, M. Pivetta, J. Dreiser, C. Piamonteze, C. P. Lutz, R. M. Macfarlane, B. A. Jones, P. Gambardella, A. J. Heinrich, and H. Brune Phys. Rev. Lett. 115, 237202 - Published 1 December 2015 DOI: 10.1103/PhysRevLett.115.237202 


\title{
Origin of perpendicular magnetic anisotropy and large orbital moment in Fe atoms on $\mathrm{MgO}$
}

\author{
S. Baumann, ${ }^{1,2}$ F. Donati, ${ }^{3}$ S. Stepanow ${ }^{4}$ S. Rusponi,${ }^{3}$ W. Paul,${ }^{1}$ S. Gangopadhyay, ${ }^{1,5}$ \\ I. G. Rau, ${ }^{1}$ G. E. Pacchioni,${ }^{3}$ L. Gragnaniello, ${ }^{3}$ M. Pivetta,${ }^{3}$ J. Dreiser,${ }^{3}{ }^{6}$ C. Piamonteze,${ }^{6}$ \\ C. P. Lutz, ${ }^{1}$ R. M. Macfarlane, ${ }^{1}$ B. A. Jones,${ }^{1}$ P. Gambardella, ${ }^{4}$ A. J. Heinrich, ${ }^{1}$ and H. Brune ${ }^{3}$ \\ ${ }^{1}$ IBM Almaden Research Center, 650 Harry Road, San Jose, CA 95120, USA \\ ${ }^{2}$ Department of Physics, University of Basel, Klingelbergstrasse 82, CH-4056 Basel, Switzerland \\ ${ }^{3}$ Institute of Condensed Matter Physics, École Polytechnique Fédérale \\ de Lausanne (EPFL), Station 3, CH-1015-Lausanne, Switzerland \\ ${ }^{4}$ Department of Materials, ETH Zürich Hönggerbergring 64, CH-8093 Zürich, Switzerland \\ ${ }^{5}$ Department of Physics, University of California, Davis CA 95616, USA \\ ${ }^{6}$ Swiss Light Source (SLS), Paul Scherrer Institute (PSI), CH-5232 Villigen PSI, Switzerland
}

\begin{abstract}
We report on the magnetic properties of individual Fe atoms deposited on $\mathrm{MgO}(100)$ thin films probed by x-ray magnetic circular dichroism and scanning tunneling spectroscopy. We show that the $\mathrm{Fe}$ atoms have strong perpendicular magnetic anisotropy with a zero-field splitting of $14.0 \pm$ $0.3 \mathrm{meV} /$ atom. This is a factor ten larger compared to the interface anisotropy of epitaxial Fe layers on $\mathrm{MgO}$ and the largest value reported for Fe atoms adsorbed on surfaces. The interplay between the ligand field at the $\mathrm{O}$ adsorption sites and spin-orbit coupling is analyzed by density functional theory and multiplet calculations, providing a comprehensive model of the magnetic properties of Fe atoms in a low-symmetry bonding environment.
\end{abstract}

The $\mathrm{Fe} / \mathrm{MgO}(100)$ interface is a fundamental building block of spintronic devices. Several key properties for the realization of magnetic tunnel junctions, such as perpendicular magnetic anisotropy (PMA) [1-4], giant tunnel magnetoresistance [5-8], and electric field control of the magnetization [9-11] are realized at once in this system. The origin of the interfacial PMA in $\mathrm{Fe} / \mathrm{MgO}$ layers has been widely discussed [2, 10-13]. According to recent first principle calculations, PMA results from a combination of both interface and "bulk" effects, in which the hybridization between Fe-3 $d$ and $\mathrm{O}-2 p$ orbitals [2], the Fe thickness [12], and the bcc-like layer stacking of the magnetic layer [13] play a substantial role. Experimental studies of the $\mathrm{Fe} / \mathrm{MgO}$ interface, however, usually start from Fe films with a thickness larger than 2-3 monolayers (ML) and uneven morphology [1,14-16], which makes it difficult to isolate purely interfacial effects and, in particular, the influence of the orbital hybridization between $\mathrm{Fe}$ and $\mathrm{MgO}$ on the magnetic moment and anisotropy.

This limitation can be overcome by studying isolated Fe atoms deposited on $\mathrm{MgO}$ thin films, for which, as it will be shown in this Letter, the magnetic properties are uniquely determined by the Fe-MgO interaction. Previous works have only focused on Fe atoms diluted in bulk $\mathrm{MgO}$ since they have long been considered as a model system for studying the interplay of crystal field, spin-orbit coupling (SOC), and magnetic moment on the low energy excitation spectra of transition-metal impurities in insulators [17-19]. However, they have never been investigated at the surface, where the local symmetry is drastically altered with respect to the octahedral environment found inside $\mathrm{MgO}$ crystals and can potentially enhance the anisotropy energy, as similarly observed in previous studies of adatoms on non-magnetic surfaces [20-28].
Here, we report a study of the magnetic properties of isolated $\mathrm{Fe}$ atoms on $\mathrm{MgO}$ thin films on $\mathrm{Ag}(100)$ performed by x-ray magnetic circular dichroism (XMCD), inelastic electron tunneling spectroscopy (IETS), multiplet calculations, and density functional theory (DFT). We show that Fe atoms preferentially bind to $\mathrm{O}$ surface sites and develop strong PMA as a result of the interplay between the low symmetry ligand field and SOC at these sites. Our IETS measurements reveal a PMA with a zero-field splitting of $14 \mathrm{meV}$ and a corresponding total anisotropy barrier of $18 \mathrm{meV} /$ atom, one order of magnitude larger with respect to the interfacial anisotropy reported in $\mathrm{Fe} / \mathrm{MgO}$ blanket layers $[1,2,13,15]$. Our analysis reveals that the first-order orbital moment of $\mathrm{Fe}$ is quenched by the weak four-fold ligand field due to the $\mathrm{Mg}$ atoms and relates the PMA to the unusually large second-order orbital moment induced by SOC at the Fe sites. These results shed light on the interfacial nature of PMA in $\mathrm{Fe} / \mathrm{MgO}$ and demonstrate the potential of $\mathrm{MgO}$ substrates to provide a low-symmetry bonding geometry for magnetic atoms, similar to that encountered in axial molecular magnets $[29,30]$.

Figure 1(a) shows an STM image of two individual Fe atoms deposited at $\approx 8 \mathrm{~K}$ on $1 \mathrm{ML} \mathrm{MgO}(100)$ grown on $\mathrm{Ag}(100)$ [31-34]. At the Fe coverages of 0.01-0.03 ML (one $\mathrm{ML}$ is defined as one $\mathrm{Fe}$ atom per $\mathrm{MgO}(100)$ unit cell) used in the present study, we observe isolated $\mathrm{Fe}$ atoms rather than clusters. We find only one Fe species with an apparent height of $180 \mathrm{pm}$. Using DFT with the generalized gradient approximation (GGA) and onsite Coulomb interactions $(U=3.2 \mathrm{eV})[35]$ for the Fe $d$-states, we find the on-top oxygen adsorption site to be the lowest in energy $[32,34]$. Figure 1 (b) reveals that the $\mathrm{O}$ beneath $\mathrm{Fe}$ is displaced by $40 \mathrm{pm}$ upwards from the 
(a)

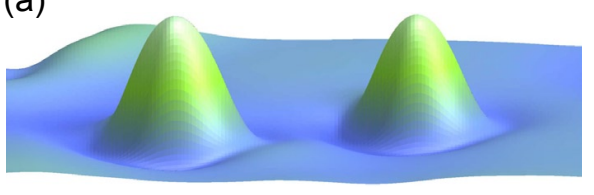

(b)

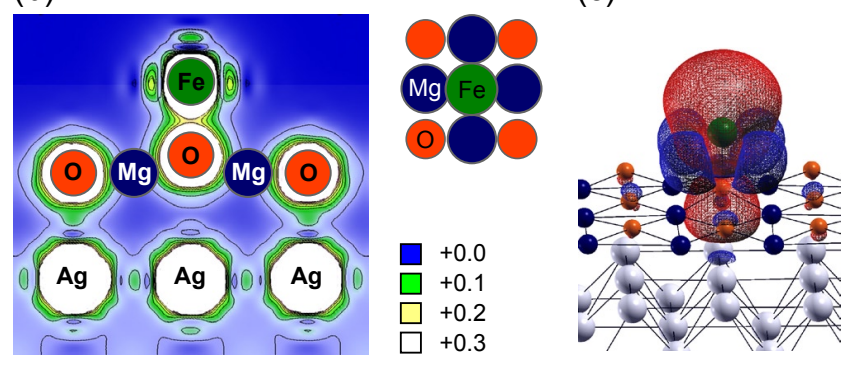

In addition, the experimental out-of-plane magnetization curve in Fig. 2(c) is well reproduced by the line showing the out-of-plane projected field-dependent total magnetic moment $\left\langle 2 S_{z}(B)\right\rangle+\left\langle L_{z}(B)\right\rangle$ derived from multiplet calculations. In these calculations we included charge transfer to the $\mathrm{O}$ ligand, leading to configuration mixing, the axial ligand field due to the nearest-neighbor $\mathrm{O}$ atom $(D s$ and $D t$ ), the cubic distortion due to the four next-nearest neighbor $\mathrm{Mg}$ atoms $(D q)$, SOC $(\zeta)$, and the external magnetic field $(B)$. Best agreement is obtained with a $90 \%$ $d^{6}+10 \% d^{7} l$ configuration of the Fe atom, where $l$ refers to a ligand hole in the neighboring $\mathrm{O}$ atom.

The configuration mixing and the axial terms $D s$ and $D t$ result in a 10-fold degenerate ground state $\left(\left\langle L_{z}\right\rangle=\right.$ $\pm 2) \otimes\left(\left\langle S_{z}\right\rangle= \pm 1.96, \pm 0.98\right.$, and 0$)[34]$. Figure 3 illustrates its evolution under the action of $D q, \zeta$, and $B$. The cubic term $D q$ strongly perturbs the lowest multiplet and creates two spin quintuplets with fully quenched orbital moments, $\left(\left\langle L_{z}\right\rangle=0\right) \otimes\left(\left\langle S_{z}\right\rangle= \pm 1.96, \pm 0.98\right.$, and $0)$. The SOC splits the lowest quintuplet with $B_{1}$ symmetry (blue) into essentially three energy levels, and restores more than half of the free-atom orbital moment by (a)

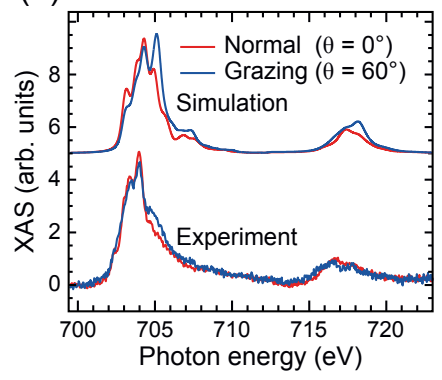

(b)

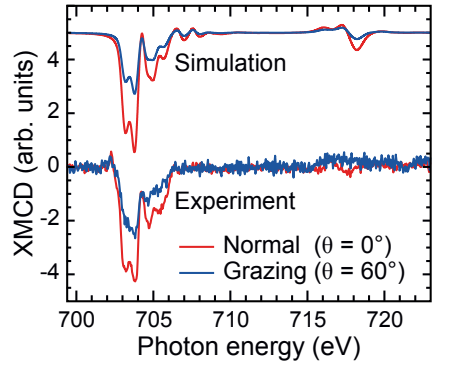

(c)

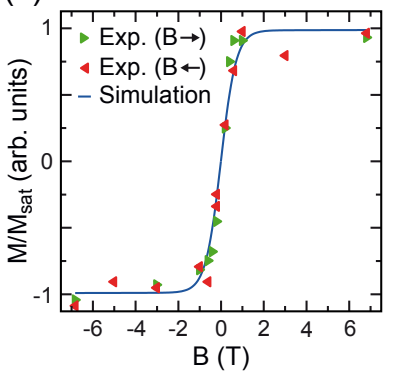

(d)

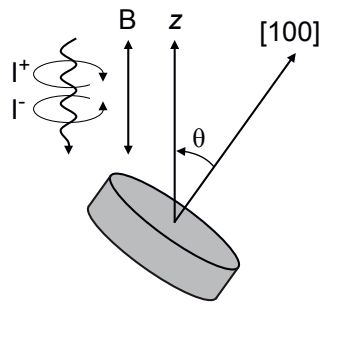

To access the magnetic properties of Fe atoms, we performed XMCD measurements at the EPFL/PSI X-Treme beamline of the Swiss Light Source [36]. X-ray absorption spectra (XAS) and the resulting XMCD signal are shown in Figs. 2(a) and (b) (see Ref. [34] for experimental details). The Fe $L_{3}$ and $L_{2}$ edges exhibit sharp multiplet structures characteristic of an ensemble of single adatoms on identical adsorption sites [37]. The XMCD signal is larger at normal than at grazing incidence, revealing a PMA in Fe atoms on $\mathrm{MgO}$.

More insight into the magnetic levels and the evolution of their energies is gained from multiplet theory [38]. The calculated spectra shown in Fig. 2 are in good agreement with the experiment for both incident beam directions.

Figure 2. (color online). (a) Measured and simulated XAS over the Fe $L_{3}$ and $L_{2}$ edges for $0.03 \mathrm{ML} \mathrm{Fe}$ on an $\mathrm{MgO}$ film on $\mathrm{Ag}(100)$ with an average thickness of $3 \mathrm{ML}(T=2.5 \mathrm{~K}$, $B=6.8 \mathrm{~T}$, incident angle $\theta$, total electron yield mode). (b) XMCD spectra for both geometries. (c) Out-of-plane magnetization curve measured by first saturating the sample at $6.8 \mathrm{~T}$ (red) and $-6.8 \mathrm{~T}$ (green) and then moving to the respective field value $(T=2.5 \mathrm{~K})$. The values of the magnetization are obtained from the maximum of the XMCD signal at $704 \mathrm{eV}$. The solid line represents $\left\langle 2 S_{z}(B)\right\rangle+\left\langle L_{z}(B)\right\rangle$ determined by the multiplet fit with a saturation moment of $5.2 \mu_{\mathrm{B}}$. (d) Sketch of the measurement geometry. The magnetic field is aligned to the incident beam. 


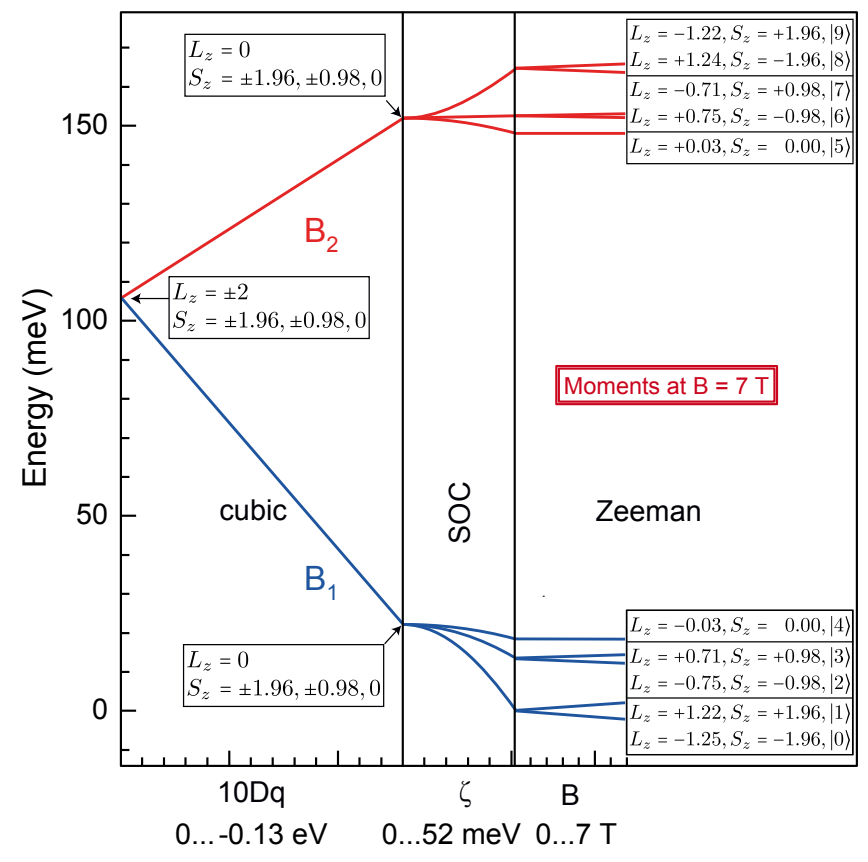

Figure 3. (color online). Energy level diagram resulting from the multiplet simulation of the XAS and XMCD spectra (for full diagram see [34]). The $S_{z}$ and $L_{z}$ values in the labels are the respective expectation values, the \langle\rangle signs have been omitted for brevity. The cubic crystal field quenches the orbital moment and creates two spin quintuplets with $B_{1}$ and $B_{2}$ symmetry, which are both further separated by the SOC and the Zeeman energy. Labels $|0\rangle-|4\rangle$ and $|5\rangle-|9\rangle$ denote the states deriving from the $B_{1}$ and $B_{2}$ quintuplets, respectively.

coupling the two lowest orbital levels in a second-order perturbation [39]. The combined effect of ligand field and SOC lowers the energy of the states with the largest $\left|S_{z}\right|$ components, and, therefore, engenders a PMA in Fe atoms.

The out-of-plane magnetic field lifts the remaining degeneracy of the five states labelled $|0\rangle-|4\rangle$. At $6.8 \mathrm{~T}$, the new ground state $|0\rangle$ exhibits large orbital $\left\langle L_{z}\right\rangle \mu_{\mathrm{B}}=$ $1.25 \mu_{\mathrm{B}}$ and spin magnetic moments $2\left\langle S_{z}\right\rangle \mu_{\mathrm{B}}=3.92 \mu_{\mathrm{B}}$, in good agreement with that obtained from DFT calculations [34]. In contrast, the excited spin quintuplet (red) has $B_{2}$ symmetry, lies $\approx 100 \mathrm{meV}$ higher in energy, and has its orbital and spin magnetic moments anti-aligned. This results in smaller total magnetic moments and hence a smaller Zeeman splitting.

Our IETS measurements on individual Fe atoms determine the energy splitting of the lowest magnetic states with high precision, and thereby complement XMCD. Figure 4(a) displays clear conductance steps with $15 \%$ amplitude located at $\pm 14.0 \pm 0.3 \mathrm{mV}$ at zero field (the error bar refers to variations between atoms at different locations of the $\mathrm{MgO}$ film). The magnetic nature of the underlying inelastic excitations is demonstrated by the splitting of the excitation energy in an out-of-plane magnetic field shown in Fig. 4(b). For in-plane fields the (a)

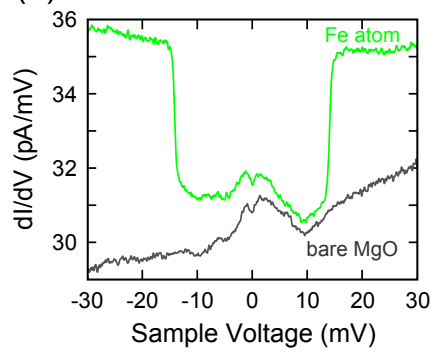

(c)

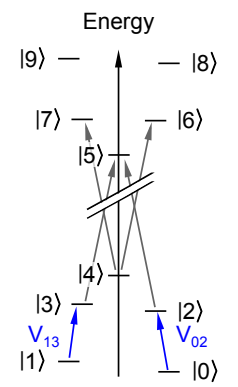

(b)

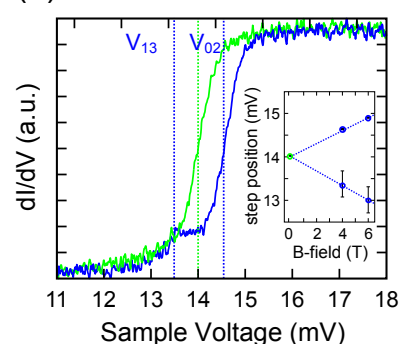

(d)

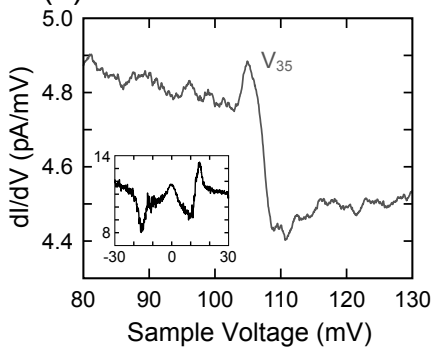

Figure 4. (color online). (a) STM differential conductance $(d I / d V)$ spectrum on $\mathrm{Fe} / 1 \mathrm{ML} \mathrm{MgO}(100) / \mathrm{Ag}(100), d I / d V$ on bare $\mathrm{MgO}$ is shown for comparison $(T=0.6 \mathrm{~K}, B=0 \mathrm{~T}$, modulation with $V_{\text {mod }}=150 \mu \mathrm{V} \mathrm{rms} \mathrm{at} f=806 \mathrm{~Hz}$, set point before opening the feedback loop $I_{\mathrm{t}}=1 \mathrm{nA}, V_{\mathrm{t}}=30 \mathrm{mV}$ ). (b) Positive conductance step at out-of-plane fields of $0 \mathrm{~T}$ (green) and $4 \mathrm{~T}$ (blue). Inset: Field splitting of step energies. (c) Sketch of the magnetic states and the allowed IETS excitations. (d) IETS feature corresponding to the superposition of $V_{35}, V_{25}, V_{47}$, and $V_{46}$, measured with a spin-polarized tip $\left(T=1.2 \mathrm{~K}, B=2 \mathrm{~T}, V_{\text {mod }}=1.5 \mathrm{mV}, I_{\mathrm{t}}=1 \mathrm{nA}, V_{\mathrm{t}}=100\right)$. Inset: spin-polarized spectrum in the same energy window as (a). Jagged edges at the conductance steps originate from the superposition of inelastic spin excitations and spin pumping, and reveal that the tip is magnetic.

splitting is absent [34], confirming the strong PMA.

Connection between the transitions excited in IETS and the states derived from the multiplet calculations is established by the level diagram shown in Fig. 4(c). The blue arrows indicate the first two excitations possible for tunnel electrons, $|0\rangle \rightarrow|2\rangle$ and $|1\rangle \rightarrow|3\rangle$, with the corresponding tunneling voltages labeled $V_{02}$ and $V_{13}$. Within the lowest multiplet, only these excitations fulfill the spin selection rule $\Delta S_{z}=0, \pm 1$ [21]. They correspond to the conductance steps in Figs. 4(a) and (b). At zero field, the value $V_{02}=V_{13}=14.0 \mathrm{mV}$ corresponds to the zerofield splitting of the ground and first excited doublets. This is in excellent agreement with the level separation of $13 \mathrm{meV}$ found in the multiplet calculations.

These excitations split symmetrically in an external out-of-plane field, see Fig. 4(b). The amplitude of $V_{13}$ is significantly smaller than that of $V_{02}$ because the corresponding transition starts from the excited state $|1\rangle$. The fact that $V_{13}$ is visible implies that the tunnel current drives the occupation of the $|1\rangle$ state, and that its lifetime is longer than the mean time between tunneling 
electrons of the order of $1 \mathrm{~ns}$ [40].

As seen from the inset of Fig. 4(b), the splitting is linear and its slope indicates an effective $g^{*}$-value of $2.57 \pm 0.06$ in the $z$-direction. This is significantly above the free-electron value of $g_{e}=2$ and thus confirms the presence of a large orbital magnetic moment for $\mathrm{Fe}$ on $\mathrm{MgO}[41,42]$. The latter evidence is independently supported by the large orbital-to-effectivespin ratio $0.71 \pm 0.08$ derived from sum-rules [34, 4345]. This value is obtained by the experimental XMCD only and even slightly exceeds the calculated ratio of 0.59 from the multiplet analysis. Although first-order unquenched orbital moments were observed in Fe-based linear molecules $[29,30]$, a value larger than $1 \mu_{\mathrm{B}} /$ atom, as observed in the present experiment, is exceptional for second-order SOC $[18,46]$.

The splitting of the lowest 5-fold multiplet, being due to the second-order SOC, can be described using a conventional spin-Hamiltonian with a quadratic anisotropy term $D S_{z}^{2}$, where $D=-4.7 \mathrm{meV}$ is the uniaxial anisotropy parameter. This approach is widely used to interpret the magnetic IETS excitations of adatoms [21, 26, 47] and molecules [48] at the surface. However, the use of STM measurements solely does not guarantee an unambiguous determination of $S$ and, therefore, of the total anisotropy barrier. In our analysis, we circumvent this issue by combining IETS and XMCD. The first measures the zero-field splitting directly and very precisely, while the second identifies the ground and excited states with their spin and orbital magnetic moments, as well as their angular anisotropies. Using the value of $S=2$ obtained from XMCD and the zero-field splitting of $14 \mathrm{meV}$ measured with IETS, we estimate a total anisotropy barrier of $\left|D S_{z}^{2}\right|=18.8 \mathrm{meV}$, in excellent agreement with the value of $18.3 \mathrm{meV}$ obtained by the energy separation between the $|0\rangle,|1\rangle$ states and the $|4\rangle$ singlet from the multiplet analysis. The total anisotropy barrier of Fe atoms on $\mathrm{MgO}$ is, therefore, strongly enhanced with respect to Fe films on $\mathrm{MgO}[1,2,13-16]$ due to the reduced in-plane coordination of the Fe atoms [23]. Remarkably, it is more than twice the largest value reported for individual Fe atoms adsorbed on other surfaces [21, 49-51] and embedded in bulk MgO [17-19], and approaches that reported for Fe atoms in linear molecules [30].

The magnetic properties of $\mathrm{Fe}$ are quite different to those of Co, which shows unquenched first-order orbital moment and record-high PMA on the $\mathrm{MgO}$ surface [28]. This is a consequence of the orbital symmetry of the corresponding magnetic states. In a four-fold symmetric ligand field, the fourth-order cubic term $D q$ combines orbital states separated by $\Delta L_{z}= \pm 4$. This allows the mixing of the Fe states with $L_{z}= \pm 2$, with the consequent formation of states with quenched first-order orbital moment. In contrast, Co has $L_{z}= \pm 3$ and is protected from such mixing, thus it exhibits a ground state orbital moment largely unchanged with respect to the free atom, together with a maximum first-order SOC splitting of $58 \mathrm{meV}$ [28].

Spin-polarized STM tips reveal an additional conductance step at $\sim 105 \mathrm{mV}$, see Fig. 4(d). Since this step is absent for non-polarized tips, we assign it to an electron driven occupation change of the magnetic states at the respective threshold energy (spin pumping). In agreement with this assignment, the step height depends on the set-point current, as the tunneling electrons must arrive frequently enough to probe the excited states before they decay [40]. According to the spin selection rule, we can assign the $\sim 105 \mathrm{mV}$ step to the four transitions $V_{35}$, $V_{25}, V_{46}$, and $V_{47}$ that are very close in energy [52]. The multiplet calculations estimate $V_{35} \approx 134 \mathrm{meV}$, in reasonable agreement with the one directly probed by STM. This confirms the validity of the multiplet model on a scale of more than $100 \mathrm{meV}$, the largest ever probed by IETS. Transitions between states belonging to different spin multiplets have been observed in spin chains $[40,53]$ and molecular magnets [54]. Unique to $\mathrm{Fe}$ on $\mathrm{MgO}$, the excitation from lower to upper spin multiplet involves a transition from aligned (lower multiplet) to anti-aligned (upper multiplet) orbital and spin moments.

In conclusion, the present study reveals how the large PMA and orbital moment of $\mathrm{Fe}$ atoms on $\mathrm{MgO}$ arise from the interplay between SOC and low-symmetry ligand field of the $\mathrm{O}$ adsorption site. Our analysis, going beyond the spin-Hamiltonian approach, allows to follow the effect of each interaction on the quantum levels of a magnetic system, and proves to be an effective approach to understand and engineer the properties of atomic-scale tunnel magnetoresistance junctions.

We acknowledge funding from the Swiss National Science Foundation, the Competence Centre for Materials Science and Technology, the COST action MP0903, DOE Stockpile Stewardship Academic Alliance Program under Grant \# DE-FG03-03NA00071, and the Office of Naval Research. We thank Bruce Melior for expert technical assistance and Claire-Lise Bandelier for preparing the figures. We used computational resources of NERSC and IBM Research Almaden in house Blue Gene $\mathrm{P}$ for our DFT calculations and data visualization.

[1] M. Klaua, D. Ullmann, J. Barthel, W. Wulfhekel, J. Kirschner, R. Urban, T. L. Monchesky, A. Enders, J. F. Cochran, and B. Heinrich, Phys. Rev. B 64, 134411 (2001).

[2] H. X. Yang, M. Chshiev, B. Dieny, J. H. Lee, A. Manchon, and K. H. Shin, Phys. Rev. B 84, 054401 (2011).

[3] S. Ikeda, K. Miura, H. Yamamoto, K. Mizunuma, H. D. Gan, M. Endo, S. Kanai, J. Hayakawa, F. Matsukura, and H. Ohno, Nat. Mater. 9, 721 (2010).

[4] M. Cubukcu, O. Boulle, M. Drouard, K. Garello, C. O. Avci, I. M. Miron, J. Langer, B. Ocker, P. Gambardella, 
and G. Gaudin, Appl. Phys. Lett. 104, 042406 (2014).

[5] J. Mathon and A. Umerski, Phys. Rev. B 63, 220403 (2001).

[6] W. H. Butler, X.-G. Zhang, T. C. Schulthess, and J. M. MacLaren, Phys. Rev. B 63, 054416 (2001).

[7] S. Yuasa, T. Nagahama, A. Fukushima, Y. Suzuki, and K. Ando, Nat. Mater. 3, 868 (2004).

[8] S. S. P. Parkin, C. Kaiser, A. Panchula, P. M. Rice, B. Hughes, M. Samant, and S. H. Yang, Nat. Mater. 3, 862 (2004).

[9] T. Maruyama, Y. Shiota, T. Nozaki, K. Ohta, N. Toda, M. Mizuguchi, A. A. Tulapurkar, T. Shinjo, M. Shiraishi, S. Mizukami, Y. Ando, and Y. Suzuki, Nat. Nanotech. 4, 158 (2009).

[10] K. Nakamura, T. Akiyama, T. Ito, M. Weinert, and A. J. Freeman, Phys. Rev. B 81, 220409 (2010).

[11] M. K. Niranjan, C.-G. Duan, S. S. Jaswal, and E. Y. Tsymbal, Applied Physics Letters 96, 222504 (2010).

[12] A. Hallal, H. X. Yang, B. Dieny, and M. Chshiev, Phys. Rev. B 88, 184423 (2013).

[13] K. Hotta, K. Nakamura, T. Akiyama, T. Ito, T. Oguchi, and A. J. Freeman, Phys. Rev. Lett. 110, 267206 (2013).

[14] C. Martínez Boubeta, C. Clavero, J. M. García-Martín, G. Armelles, A. Cebollada, L. Balcells, J. L. Menéndez, F. Peiró, A. Cornet, and M. F. Toney, Phys. Rev. B 71, 014407 (2005).

[15] C.-H. Lambert, A. Rajanikanth, T. Hauet, S. Mangin, E. E. Fullerton, and S. Andrieu, Applied Physics Letters 102, 122410 (2013).

[16] J. Okabayashi, J. W. Koo, H. Sukegawa, S. Mitani, Y. Takagi, and T. Yokoyama, Applied Physics Letters 105, 122408 (2014).

[17] F. S. Ham, W. M. Schwarz, and M. C. M. O'Brien, Phys. Rev. 185, 548 (1969).

[18] T. Haupricht, R. Sutarto, M. W. Haverkort, H. Ott, A. Tanaka, H. H. Hsieh, H. J. Lin, C. T. Chen, Z. Hu, and L. H. Tjeng, Phys. Rev. B 82, 035120 (2010).

[19] A. Ferrón, F. Delgado, and J. Fernández-Rossier, N. J. Phys. 17, 033020 (2015).

[20] P. Gambardella, S. Rusponi, M. Veronese, S. S. Dhesi, C. Grazioli, A. Dallmeyer, I. Cabria, R. Zeller, P. H. Dederichs, K. Kern, C. Carbone, and H. Brune, Science 300, 1130 (2003).

[21] C. F. Hirjibehedin, C. Y. Lin, A. F. Otte, M. Ternes, C. P. Lutz, B. A. Jones, and A. J. Heinrich, Science 317, 1199 (2007).

[22] F. Meier, L. Zhou, J. Wiebe, and R. Wiesendanger, Science 320, 82 (2008).

[23] H. Brune and P. Gambardella, Surf. Sci. 603, 1812 (2009).

[24] S. Loth, S. Baumann, C. P. Lutz, D. M. Eigler, and A. J. Heinrich, Science 335, 196 (2012).

[25] A. A. Khajetoorians, B. Baxevanis, C. Hübner, T. Schlenk, S. Krause, T. O. Wehling, S. Lounis, A. Lichtenstein, D. Pfannkuche, J. Wiebe, and R. Wiesendanger, Science 339, 55 (2013).

[26] F. Donati, Q. Dubout, G. Autès, F. Patthey, F. Calleja, P. Gambardella, O. V. Yazyev, and H. Brune, Phys. Rev. Lett. 111, 236801 (2013).

[27] F. Donati, L. Gragnaniello, A. Cavallin, F. D. Natterer, Q. Dubout, M. Pivetta, F. Patthey, J. Dreiser, C. Piamonteze, S. Rusponi, and H. Brune, Phys. Rev. Lett. 113, 177201 (2014).

[28] I. G. Rau, S. Baumann, S. Rusponi, F. Donati,
S. Stepanow, L. Gragnaniello, J. Dreiser, C. Piamonteze, F. Nolting, S. Gangopadhyay, O. R. Albertini, R. Macfarlane, C. P. Lutz, B. A. Jones, P. Gambardella, A. J. Heinrich, and H. Brune, Science 344, 988 (2014).

[29] W. M. Reiff, A. M. LaPointe, and E. H. Witten, J. Am. Chem. Soc. 126, 10206 (2004).

[30] J. M. Zadrozny, D. J. Xia, M. Atanasov, G. J. Long, F. Grandjean, F. Neese, and J. R. Long, Nat. Chem. 5, 577 (2013).

[31] S. Schintke, S. Messerli, M. Pivetta, F. Patthey, L. Libioulle, M. Stengel, A. De Vita, and W. D. Schneider, Phys. Rev. Lett. 87, 276801 (2001).

[32] K. Neyman, C. Inntam, V. Nasluzov, R. Kosarev, and N. Rösch, Appl. Phys. A 78, 823 (2004).

[33] S. Baumann, I. G. Rau, S. Loth, C. P. Lutz, and A. J. Heinrich, ACS Nano 8, 1739 (2014).

[34] See Supplementary Information, which includes Refs. [56-71], for details about the sample preparation, the STM and XMCD measurements, and the DFT and multiplet calculations.

[35] M. Cococcioni and S. de Gironcoli, Phys. Rev. B 71, 035105 (2005).

[36] C. Piamonteze, U. Flechsig, S. Rusponi, J. Dreiser, J. Heidler, M. Schmidt, R. Wetter, M. Calvi, T. Schmidt, H. Pruchova, J. Krempasky, C. Quitmann, H. Brune, and F. Nolting, J. Synchrotron Rad. 19, 661 (2012).

[37] P. Gambardella, S. S. Dhesi, S. Gardonio, C. Grazioli, P. Ohresser, and C. Carbone, Phys. Rev. Lett. 88, 047202 (2002).

[38] F. de Groot, Chem. Rev. 101, 1779 (2001).

[39] B. McGarvey, in Transition Metal Chemistry, Vol. 3, edited by R. L. Carlin and M. Dekker (John Wiley \& Sons, New York, 1966).

[40] S. Loth, K. von Bergmann, M. Ternes, A. F. Otte, C. P. Lutz, and A. J. Heinrich, Nat. Phys. 6, 340 (2010).

[41] B. Chilian, Phys. Rev. B 84, 212401 (2011).

[42] T. Schuh, T. Balashov, T. Miyamachi, S. Y. Wu, C. C. Kuo, A. Ernst, J. Henk, and W. Wulfhekel, Phys. Rev. B 84, 104401 (2011).

[43] B. T. Thole, P. Carra, F. Sette, and G. van der Laan, Phys. Rev. Lett. 68, 1943 (1992).

[44] P. Carra, B. T. Thole, M. Altarelli, and X. Wang, Phys. Rev. Lett. 70, 694 (1993).

[45] The ratio is defined as $\left\langle L_{z}\right\rangle /\left\langle 2 S_{z}+7 T_{z}\right\rangle$, where $\left\langle T_{z}\right\rangle$ is the $z$-projected dipolar magnetic moment.

[46] J. Bartolomé, F. Bartolomé, L. M. García, G. Filoti, T. Gredig, C. N. Colesniuc, I. K. Schuller, and J. C. Cezar, Phys. Rev. B 81, 195405 (2010).

[47] A. F. Otte, M. Ternes, K. von Bergmann, S. Loth, H. Brune, C. P. Lutz, C. F. Hirjibehedin, and A. J. Heinrich, Nat. Phys. 4, 847 (2008).

[48] N. Tsukahara, K.-i. Noto, M. Ohara, S. Shiraki, N. Takagi, Y. Takata, J. Miyawaki, M. Taguchi, A. Chainani, S. Shin, and M. Kawai, Phys. Rev. Lett. 102, 167203 (2009).

[49] A. A. Khajetoorians, S. Lounis, B. Chilian, A. T. Costa, L. Zhou, D. L. Mills, J. Wiebe, and R. Wiesendanger, Phys. Rev. Lett. 106, 037205 (2011).

[50] A. A. Khajetoorians, T. Schlenk, B. Schweflinghaus, M. dos Santos Dias, M. Steinbrecher, M. Bouhassoune, S. Lounis, J. Wiebe, and R. Wiesendanger, Phys. Rev. Lett. 111, 157204 (2013).

[51] G. E. Pacchioni, L. Gragnaniello, F. Donati, M. Pivetta, G. Autès, O. V. Yazyev, S. Rusponi, and H. Brune, Phys. 
Rev. B 91, 235426 (2015).

[52] Note that $\Delta S_{z}= \pm 1,0$ also allows the $|0\rangle \rightarrow|6\rangle$ transition. This transition has a higher energy. The fact that it is not giving a significant contribution to the $d I / d V$ spectrum signifies that its rate is low and that the excited states are long-lived such that we are not limited to transitions starting from the ground state.

[53] C. F. Hirjibehedin, C. P. Lutz, and A. J. Heinrich, Science 312, 1021 (2006).

[54] S. Kahle, Z. Deng, N. Malinowski, C. Tonnoir, A. Forment-Aliaga, N. Thontasen, G. Rinke, D. Le, V. Turkowski, T. S. Rahman, S. Rauschenbach, M. Ternes, and K. Kern, Nano Lett. 12, 518 (2012).

[55] A. J. Heinrich, J. A. Gupta, C. P. Lutz, and D. M. Eigler, Science 306, 466 (2004).

[56] R. C. Jaklevic and J. Lambe, Phys. Rev. Lett. 17, 1139 (1966).

[57] J. S. Griffith, Phys. Rev. 132, 316 (1963).

[58] P. Giannozzi, S. Baroni, N. Bonini, M. Calandra, R. Car, C. Cavazzoni, D. Ceresoli, G. L. Chiarotti, M. Cococcioni, I. Dabo, A. D. Corso, S. de Gironcoli, S. Fabris, G. Fratesi, R. Gebauer, U. Gerstmann, C. Gougoussis, A. Kokalj, M. Lazzeri, L. Martin-Samos, N. Marzari, F. Mauri, R. Mazzarello, S. Paolini, A. Pasquarello, L. Paulatto, C. Sbraccia, S. Scandolo, G. Sclauzero, A. P. Seitsonen, A. Smogunov, P. Umari, and R. M. Wentzcovitch, J. Phys. Condens. Matter 21, 395502 (2009).

[59] P. Blaha, K. Schwarz, G. Madsen, D. Kvasnicka, and J. Luitz, WIEN2k: An augmented plane wave+ local or- bitals program for calculating crystal properties. (Karlheinz Schwarz, Techn. Universitat Wien, Austria, 2001).

[60] M. Sgroi, C. Pisani, and M. Busso, Thin Solid Films 400, 64 (2001).

[61] B. D. Yu, Phys. Rev. B 71, 193403 (2005).

[62] O. R. Albertini, A. Y. Liu, and B. A. Jones, Phys. Rev. B 91, 214423 (2015).

[63] M. Brooks, Physica B+C 130, 6 (1985).

[64] O. Eriksson, B. Johansson, R. C. Albers, A. M. Boring, and M. S. S. Brooks, Phys. Rev. B 42, 2707 (1990).

[65] C. O. Rodriguez, M. V. Ganduglia-Pirovano, E. L. Peltzer y Blancá, M. Petersen, and P. Novák, Phys. Rev. B 63, 184413 (2001).

[66] A. Lehnert, S. Rusponi, M. Etzkorn, S. Ouazi, P. Thakur, and H. Brune, Phys. Rev. B 81, 104430 (2010).

[67] C. T. Chen, Y. U. Idzerda, H.-J. Lin, N. V. Smith, G. Meigs, E. Chaban, G. H. Ho, E. Pellegrin, and F. Sette, Phys. Rev. Lett. 75, 152 (1995).

[68] S. Stepanow, A. Mugarza, G. Ceballos, P. Moras, J. C. Cezar, C. Carbone, and P. Gambardella, Phys. Rev. B 82, 014405 (2010).

[69] G. van der Laan and B. T. Thole, Phys. Rev. B 43, 13401 (1991).

[70] O. Gunnarsson and K. Schönhammer, Phys. Rev. B 28, 4315 (1983).

[71] A. Abragam and B. Bleaney, Electron Paramagnetic Resonance of Transition Ions (Clarendon Press, Oxford, 1986). 